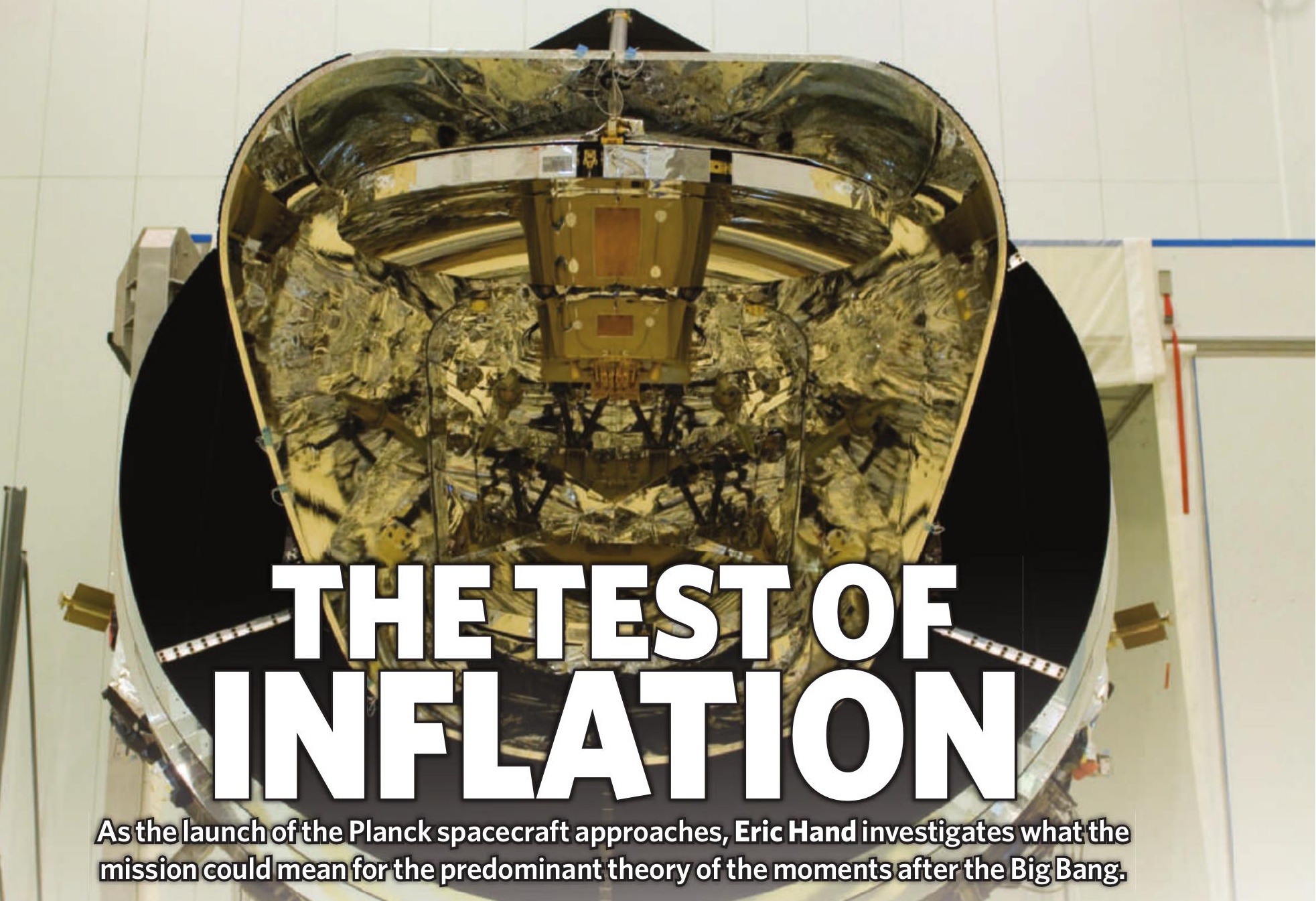

pace is cold. But Planck will be even colder. At the heart of the European Space Agency spacecraft, chilled to 0.1 kelvin by the most sophisticated cryogenic system ever put into space, a collection of gossamer threads will be suspended in near vacuum. These threads, looking like an array of spiderwebs, will gather photons from the cosmic microwave background (CMB) - the afterglow of the Universe's creation.

The extreme cold will be necessary to fulfil Planck's mission to make ultraprecise maps of the CMB. Primordial photons, the oldest light in the Universe, stream through every cubic centimetre of empty space at an average temperature of just $2.7 \mathrm{kelvin}$. The temperature of the photons will vary very slightly, depending on which part of the sky they are coming from (see 'Mapping the cosmos', opposite).

Planck's supercold detectors are designed to measure these differences in temperature at the level of less than a millionth of a kelvin (see 'Cooling Planck down', page 823). That extraordinary precision, say the spacecraft's designers, means that once launched - a milestone now scheduled for 6 May - the probe could over its 2-year lifetime settle questions that have roiled the astrophysical community for a generation. "We have to dig deeper and Planck is our next best chance to do that," says project scientist Jan Tauber, based in Noordwijk, the Netherlands.

For nearly 30 years, says Tauber, the thinking of cosmologists has been guided by a theory called inflation, which tries to explain how the Universe evolved in the moments after the Big Bang. Inflation has passed every observational test to date, mainly by predicting the statistics of the temperature variations in the CMB seen by Planck's predecessors. But Planck's extreme sensitivity to the variations will put inflation to its most stringent test yet - and will either vindicate it, or demolish it in favour of some rival theory, of which there are several.

Indeed, inflation is so important to modern cosmology that the ultracool, 2-tonne, €600-million (US\$-800 million) spacecraft is in a very hot competition with dozens of ground-based and balloon-borne experiments, all pursuing the same goal: exquisite measurements of the CMB (see 'The race for B-modes', page 824 ). "This is a very big race," says Michael Turner, a cosmologist at the University of Chicago in Illinois. "This is Swedish gold."

\section{A helping hand}

The Big Bang would have stayed pretty small without some sort of boost. To reconcile quantum theory with cosmology, physicists would like to believe that the primordial Universe started out just $10^{-35}$ metres across. That leads to a contradiction. The age of the Universe is reasonable well known: 13.7 billion years. If it started off as small as the theorists would like and expanded only at the rate that now prevails, it would still be able to fit comfortably within the full stop at the end of this sentence.

Inflation explains this apparent paradox by postulating a spectacular expansion in the very first moments (see 'Timeline of the inflationary Universe', page 822). There is no easy analogy for how furious and quick an expansion it was, but as an example, in one simple inflation model, that same infinitesimal Universe could have spread to something like $10^{1,000,000,000,000}$ metres across. That's a one followed by a trillion zeros. And it would have done so in 
a trillionth of a trillionth of a trillionth of a second. Not even light could keep up: the farthest a photon could have travelled since the Big Bang - a scale known as the horizon distance - is 'only' about $10^{27}$ metres. (This is not a contradiction with relativity: no two particles located at the same point during inflation ever had a relative speed greater than light. The explosive speed refers instead to the scale of the Universe as a whole.)

In addition to explaining the Universe's colossal size, inflation neatly solves many other problems. It explains why the Universe appears geometrically flat rather than curved - think of a balloon that has been blown up so far that its surface looks like an infinite plane. And it explains how a Universe that easily could have looked utterly different in every direction in fact looks pretty much the same, with the same average density of galaxies and the same average CMB temperature.

On top of all that, inflation explains the galaxies of which we are a part. Although it made the infant Universe almost completely flat and uniform, inflation also had to obey the dictates of quantum mechanics, which produced the tiniest of fluctuations in density from point to point. So some parts of the Universe would have ended up denser than others. These denser regions would have become the seeds around which galaxies and stars would gravitationally coalesce. And earlier observatories such as NASA's Wilkinson Microwave Anisotropy Probe (WMAP), which was launched in 2001, have shown that these fluctuations in the CMB not only exist, but have precisely the kind of size distribution predicted by inflation ${ }^{1}$.

\section{Naming the unknown}

Yet for all its explanatory power, inflation has its problems. For starters, no one knows what did the inflating. Theorists describe the 'force' as a field and give it a name - the inflaton - but the mystery remains. It is the same frustration that bedevils astronomers studying dark energy, an unknown force that accounts for three-quarters of the energy in the Universe and still accelerates its expansion. Could the cause of inflation also be the driver behind dark energy? It is an interesting similarity, but they act on vastly different scales; dark energy is a flea to inflation's elephant. "It seems unlikely that they're related," says Turner. "Which is a good reason to pursue that idea," he adds impishly.

A bigger problem for inflation, according to Paul Steinhardt, a physicist at Princeton University in New Jersey, is not so much what it is, but how it stopped. "Once it starts it never ends," says Steinhardt, who was one of inflation's founding fathers in the 1980s, but is now one of its chief critics. There is no obvious reason why the ultrarapid expansion should ever slow down to the much more modest rates of expansion seen today. "At first," says Steinhardt sarcastically, "this was celebrated."

The 'celebration' of this notion of inflation without brakes is a jab at Steinhardt's colleague and sometimes rival Andrei Linde: a physicist at Stanford University in California. (Linde, Steinhardt and Steinhardt's graduate student of the early 1980s often share in the credit given to Alan Guth, now at the Massachusetts Institute of Technology in Cambridge, for originating the theory that Guth first set out in 1980 (ref. 2).)

By 1986, Linde theorized that, because of quantum fluctuations, some portions of the Universe might feel the inflationary force more strongly than the average portion ${ }^{3}$. The result in each case would be a localized bulge. Because of inflation's immense strength, however, the bulge would quickly take over and inflate into a whole new universe,

\section{Mapping the cosmos}

When the cosmic microwave background (CMB) was first detected in the 1960s, it was just a uniform hum on the sky. But a series of spacecraft, built for precision cosmology, has revealed the $\mathrm{CMB}$ in ever-increasing detail. "Last year's discovery is this year's calibration," says Nobel prizewinner George Smoot, who was a principal investigator for COBE. Shown here are three important spacecraft and sample allsky maps (simulated for Planck).

\section{Cosmic Background Explorer (COBE): 1989 launch}

COBE saw temperature differences at large angular scales by comparing big patches of sky. This anisotropy - at levels of 1 part in 100,000 or about 30 microkelvin contained the seeds of galaxies.

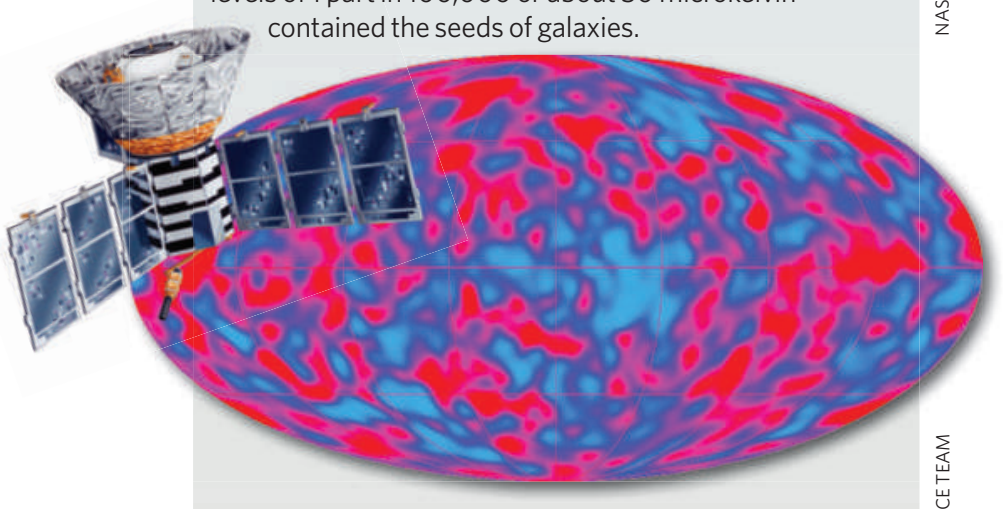

Wilkinson Microwave Anisotropy Probe (WMAP): 2001 launch

WMAP maps at much smaller angular scales. It has 45 times the sensitivity and 33 times the angular resolution of COBE. Still operating, WMAP has provided, among other things, the best estimates for the age and composition of the Universe.

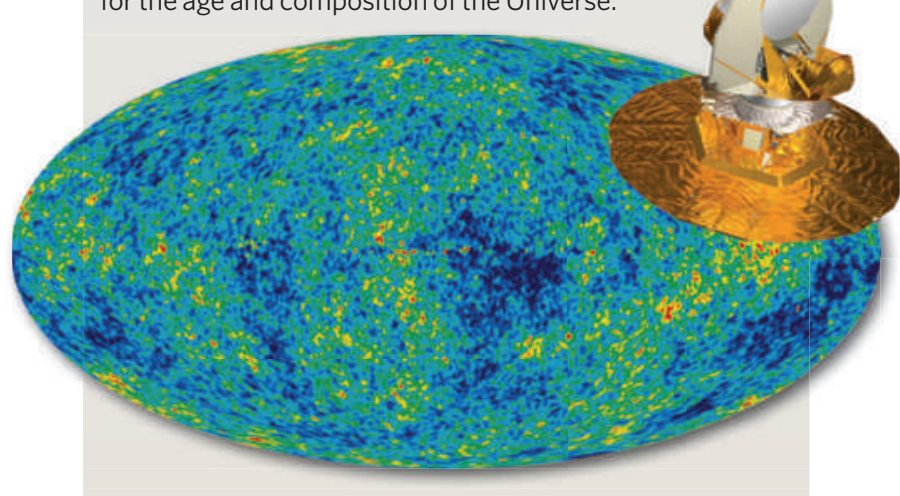

Planck: 2009 launch

Planck will be the first CMB spacecraft to carry bolometers - ultrasensitive thermometers. Planck will have more than 10 times the sensitivity and 3 times the angular resolution of WMAP, giving it a chance to find indirect evidence for gravitational waves. 


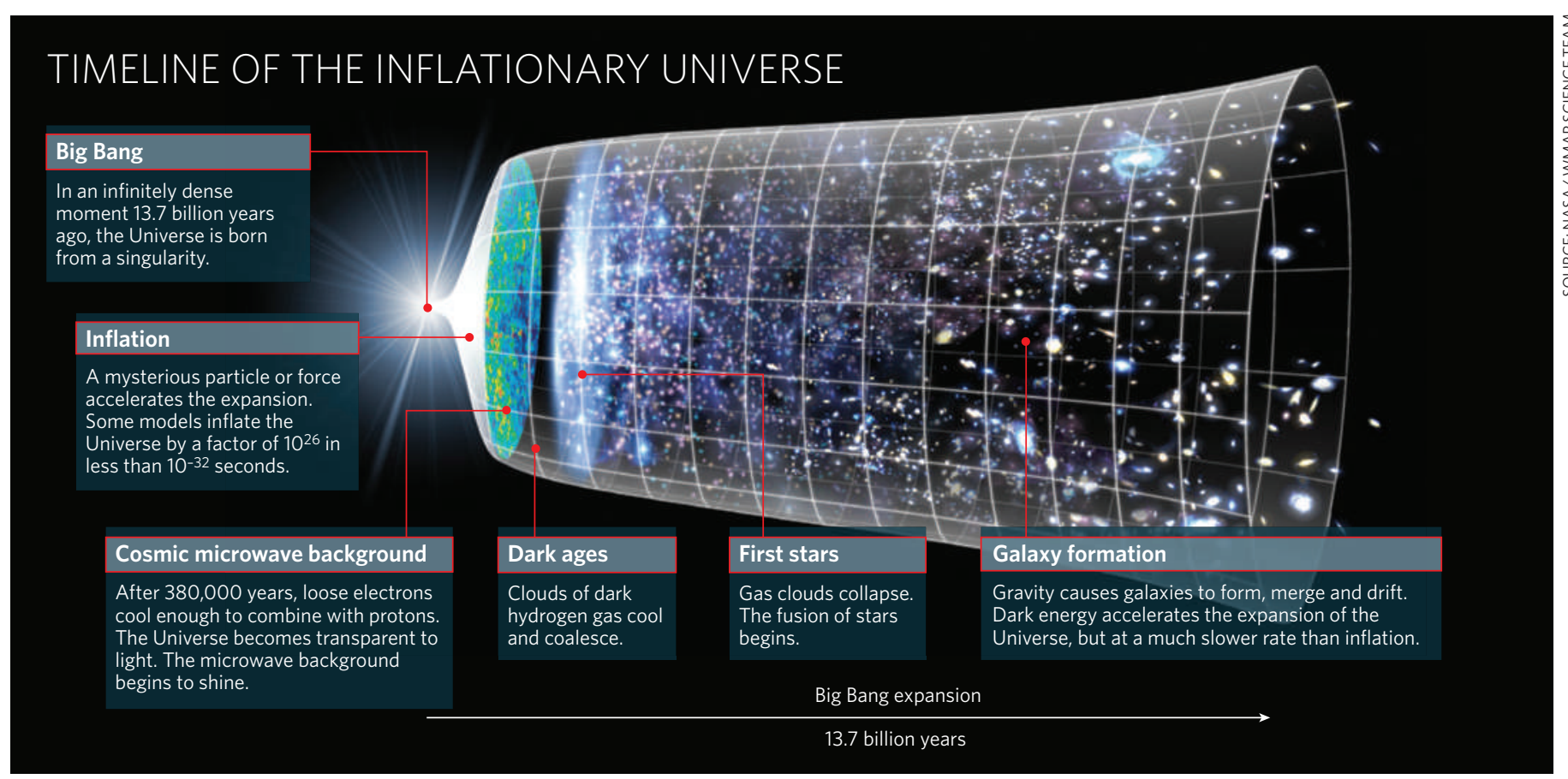

which would be attached to the old one by nothing more than a quantum-scale thread. To residents of the old universe, that connection would be too small to see; they would never know that a new creation had happened right in front of them. To residents of the new universe, it would look like a new Big Bang of their own. Indeed, universe could sprout on universe, like a fractal. The multiverse, as Linde calls it, would go on forever.

But where Linde saw something creative, an endlessly budding tree, Steinhardt saw something more like an aneurysm, debilitating if not deadly to the overarching theory. If the new offshoot universes tend to take over, the ones left behind become rare islands. What you think is going to be a typical universe is quickly left behind by newer, emerging universes. Conceivably, moreover, the physical laws could change with each emergence, so theorists couldn't know if the physics seen in our Universe governs overall. "The part that we observe - our Universe - exists almost nowhere. It's the rare region," Steinhardt says.

There is a third issue with inflation - less a problem of the theory itself than an issue of its reach or scope. As it stands, inflation is not a theory of how it all began, but a theory of how it all began just after the beginning. Call it a morning-after theory.

In inflation, the Big Bang itself remains an unknowable, infinitely dense moment of time and space called a singularity. "The biggest weakness is the notion that we can get by in a theory of cosmology without understanding the singularity," says Neil Turok, the director of the Perimeter Institute for Theoretical Physics in Waterloo, Canada. "It's like, 'let's just start the clock a little bit afterwards'."

Steinhard points out that the term Big Bang was originally coined by Fred Hoyle, a staunch opponent of the idea, as a way of mocking the notion of a cosmos suddenly appearing in the clap of a magician's hands. Ironically, the term stuck. "Some people like the idea of there being a moment of creation," Steinhardt says.

But he isn't one of them. Nor is Turok. In 2001, the two physicists proposed a radical alternative to inflation called ekpyrosis, from the Greek for 'out of fire' ${ }^{\text {. It grew }}$ out of discussions with string theorists, who see the visible world as inhabiting lower-dimensional membranes,

or branes, in a universe made up of at least 10 dimensions. Steinhardt and Turok proposed two universes on separate three-dimensional branes that would oscillate back and forth along a mutually perpendicular dimension, like sheets hung out to dry on parallel washing lines. Every trillion years or so, after each universe had dissipated into darkness during an expansive phase, the two branes would approach one another and collide, releasing a fireball of energy to start each universe afresh. "It would mean that the Big Bang wouldn't be a beginning but a collision," says Steinhardt.

\section{Avoiding the singularity}

Ekpyrosis mimics many of inflation's appealing features, with some key differences. Almost by definition, it avoids the singularity, because it describes the collision of branes

"There are people who like making a mess, and there are people who like cleaning it up." - David Spergel as a continuous process. "We have to resolve stuff from before to after," says Turok. "Inflation can get away with ignoring it." Turok says string theory has given him tools that now allow him to describe, through ekpyrosis, a crunch that avoids a singularity altogether.

Linde says he will believe it when he sees it. He has pointed out mathematical problems in ekpyrosis, and forced its proponents to revise their thinking since their original proposal. He occasionally enlists the help of string theorists, but he says he now has a hard time getting them to pay any attention to inflation's alternatives. If anything, the big advance in the past five years is that string theorists are finally finding linkages between their work and inflation. Ekpyrosis, says Linde, is "like a house of cards".

Most theorists would say that inflation is still the best game in town. But Daniel Baumann, a postdoctoral researcher at Harvard University who has worked with Steinhardt and Turok, and who is the lead author on a recent paper that maps out the theoretical landscape of inflation and its alternatives ${ }^{5}$, speaks for many when he adds that it is also "something that should be challenged".

Generational politics might be playing a role in the debate, says Linde. Young scientists don't want a career based on polishing an existing theory, he says. They would rather strike out on their own with something revolutionary - as he did when he first published on inflation in his early $30 \mathrm{~s}$ 


\section{Cooling Planck down}

The Planck spacecraft, once it settles in a gravitational dead spot 1.5 million kilometres from Earth, will face away from the Sun. "No sunshine ever falls on the core," says Charles Lawrence, the US project scientist for the mission at the Jet Propulsion Laboratory in Pasadena, California. "No earthshine. No moonshine. It's completely in the dark." And it must stay incredibly cold to have any chance of detecting the tiny temperature differences between photons.

A passive system of three stacked disks starts the elaborate process of dissipating the heat from Planck's posterior, which will face the Sun and reach 380 kelvin. Working like a car radiator, the disks spew heat away, and bring the temperature down to 50 kelvin.

Once Planck reaches orbit, three cryogenic coolers, nested like Russian dolls, will turn on. The first one, using the heat-sucking ability of decompressed hydrogen, drops the temperature to 20 kelvin. The second, a mechanical compressor, sends temperatures plummeting to 4 kelvin. And the final cooler, which uses the different thermal capacity of helium and a helium isotope with one less neutron, brings molecular movement nearly to a standstill: a tenth of a degree above absolute zero.

In this innermost sanctum lie 52 bolometers, which take the energy of electromagnetic radiation - cosmic microwaves in this case - and convert it into heat that is measured by a thermometer. The bolometers are made from fibres of gold-coated silicon nitride, a hundredth the width of a human hair. They hang in place like the filigree web of a spider: mostly empty space to allow cosmic rays, or charged particles, to pass through without confounding measurements. The spindly threads are the right size, however, to react to cosmic microwave background radiation. Corrugated antennas that poke through the nested cryocoolers gather microwaves at different frequencies and funnel them to the bolometers.

It's mind boggling, says Lawrence. Planck will gather photons that have been moving since the Big Bang and show if some are a ten-millionth of a degree hotter than others. "It's not the movie, it's not the historical novel, these are the original photons," says Lawrence. "They've been travelling 13.7 billion years to us. I think about that all the time."

E.H.

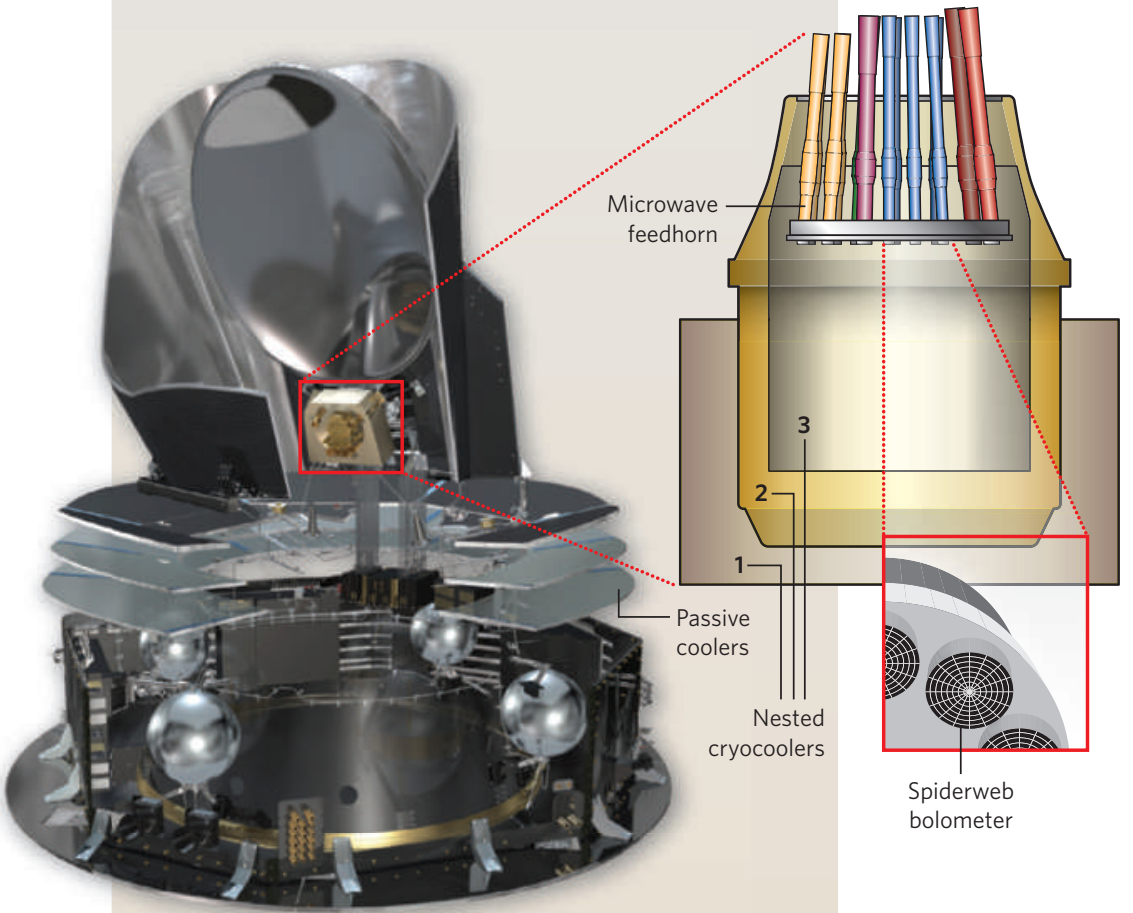

- whereas those who erected the framework of inflation have a vested interest in defending it. But that doesn't explain the iconoclasm of older scientists such as Turok, and especially Steinhardt, who helped establish inflation. With them, according to David Spergel, an astrophysicist at Princeton University who is on the WMAP team, it is just a matter of personal style. "There are people who like making a mess," he says, "and there are people who like cleaning it up."

\section{Variations on a theme}

There could be another explanation for the restlessness among theorists. They may just be bored with a lack of new data. The WMAP data have nearly been wrung dry. Existing CMB data somewhat constrain the development of new theories, which is especially challenging if you want to invent a radical one like ekpyrosis. But there are few barriers to creating a new flavour of inflation. Variations on the theme are proposed (and often, quickly quashed) all the time: such as racetrack inflation, multi-field inflation and hyperextended inflation. Each of them satisfies the basic tenets of inflation, but the differences - primarily the shape and duration of the inflationary pulse - cannot be distinguished with existing data.

Planck could change that situation. Two tests will be particularly important, not only in weeding out the imposters, but in constraining key parameters such as when inflation began — if it happened at all — and how long it lasted. One of these tests will be for the 'Gaussianity' of the CMB. The mottled temperature variations of the CMB sky seem random, but the likelihood of a hot spot sitting next to a cool spot may follow some rules. If a perfect and simple inflation governed the early Universe, then these shifts from hot to cold - the variations of the variations should have the Gaussian shape of a bell curve. Ekpyrosis, and many of the more complicated versions of inflation, have characteristic deviations from the Gaussian curve. "The implications of non-Gaussianity are so profound, you really want evidence that it's there," says Spergel.

Last year, using WMAP data, Benjamin Wandelt, a Planck scientist from the University of Illinois at UrbanaChampaign, concluded to his own surprise that the CMB seemed to have significant non-Gaussianity ${ }^{6}$. But the result, based on months of intensive computer time, could easily evaporate even though it's supposed to be $99.5 \%$ certain. "It's at the level of being tantalizing but not conclusive," says Wandelt. The WMAP team, which has performed analyses of its own, has so far resisted a declaration of non-Gaussianity. But the number of studies claiming non-Gaussianity continues to grow, including a recent one led by Christoph Räth of the Max Planck Institute for Extraterrestrial Physics in Garching, Germany". "There's a consistent picture of inconsistencies," he says. Without Planck, however, the existing hints are unlikely to congeal.

A bigger test for inflation, the 'smoking gun' that has scientists building microwave telescopes at the South Pole and sending balloons nearly to space, is the search for B-modes. B-modes are a special type of polarization that may be present as markings on the CMB. If they exist, they would be indirect evidence for the gravitational waves that should have accompanied inflation.

Ekpyrosis is clear about this: it predicts no gravitational waves whatsoever, and so B-modes should be absent. With inflation, the B-mode story, like the prospects for Gaussianity, is more complex. The simplest form of inflation should produce a relatively large B-mode signal, just within reach of Planck. But in other forms of inflation, the signal could be tiny. If Planck detects a B-mode signal and 


\section{The race for $\mathrm{B}$-modes}

Gravitational waves right after the Big Bang may have tattooed the cosmic microwave background (CMB) with signals that would be evidence for inflation. "It would be an almost guaranteed Nobel prize to whatever group was able to detect this," says James Hinderks, a postdoctoral researcher at Goddard Space Flight Center in Greenbelt, Maryland.

New generations of ground-based, balloon-borne and satellite experiments are vying to be the first to claim a detection of the signals, called B-modes, which would be a measure of variations in the way the $\mathrm{CMB}$ is polarized across the sky. But B-modes don't definitely exist. And if they do, they are at least a thousand times fainter than the CMB. A simple inflation model would put B-modes within the range of most experiments - but other inflationary theories set a much lower bar.

Hinderks is working on one of the experiments, the Primordial Inflation Polarization Explorer (PIPER) that, if it flies in 2013, would be an order of magnitude more sensitive to B-modes than Planck. But to do that, he'll have to lift a 3,500-litre tub of liquid helium with a stratospheric balloon. A microwave telescope would peer out of the bucket towards the 'southern hole' - a small window in the sky, nearly free of dust from the Milky Way, with a clear shot all the way to the CMB. Groundbased telescopes in Antarctica would do the same thing during long polar winters - when the atmosphere is extraordinarily dry and free of water vapour, which emits microwave radiation. In space, Planck will eliminate the confounding effect of foreground microwaves and look for a B-mode blip at much larger scales on the sky. The Planck team plans to analyse and release its first data set by the end of 2012, by which time some of the other experiments could have results.

There are trade-offs in each approach. Ground-based telescopes can be built cheaply with the latest technology; satellites are expensive and slow to develop, but are able to support clear and wide views of the sky. Balloons occupy a middle ground. Total US research funding for a dozen suborbital CMB experiments is about

US $\$ 25$ million a year, much less than any space-based successor to Planck, which would cost many hundreds of millions of dollars. "\$25 million is peanuts," says Stephan Meyer, a physicist at the University of Chicago in Illinois, "and the science we get out of this is fabulous."

But regardless of the observation post, all will have to make very precise temperature measurements: the millionths and even billionths of a degree differences between Big Bang photons from one part of the sky to another. "You're talking about measurements of temperature variations at the nano-Kelvin level," says Michael Turner of the University of Chicago in Illinois. "That's how important this [work] is, that people would worry about [doing] this."
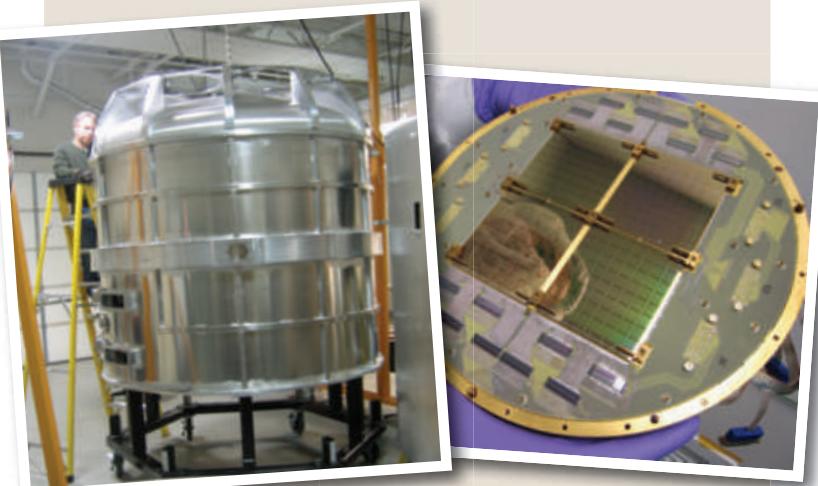

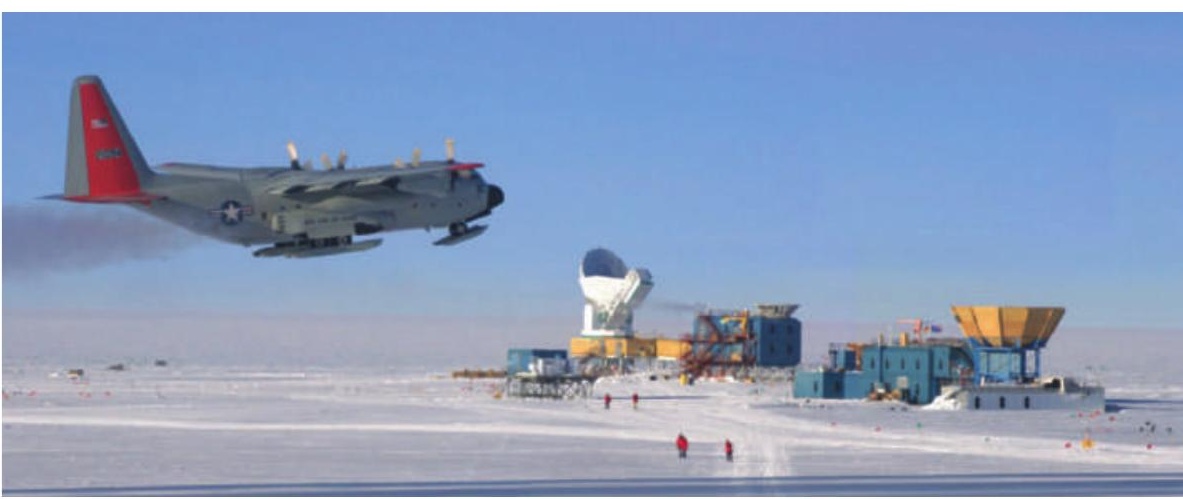

In Antarctica: BICEP (on the central blue building) looks for B-modes from the ground.

non-Gaussianity, inflation would certainly be "in more of a corner", according to Turok. Unable to resist a poke, he adds: "It's already in a corner."

A non-detection of B-modes by Planck would put the pressure on the ground-based and balloon experiments, some of which claim to have B-mode sensitivities about an order of magnitude better, although they cover far less of the sky. But Andrew Lange, a physicist at the California Institute of Technology in Pasadena, says there are limits to how far they can go. Lange has probably done more than anyone to pioneer the technology of bolometers, high-resolution microwave thermometers that will be used on Planck. If the B-mode signal is too weak, then the contemporary microwave emissions of the Universe (mostly from the Milky Way) will drown out the relic signal no matter how good the bolometer.

At that point, the only opportunity to study inflation would be to observe the gravitational waves directly, via a mission such as the Big Bang Observer. A proposed successor to the Laser Interferometer Space Antenna - another far-off space mission to detect and measure gravitational waves - the Big Bang Observer would have the tough task of directly detecting the low hum of inflation's gravitational waves, rather than picking them up indirectly in the $\mathrm{CMB}$, as the $\mathrm{B}$-mode experiments try to. But the Big Bang Observer's technology, and its funding, are still just theoretical. And without hints of a signal from one of the B-mode experiments, the project will be hard to justify. If the bang was indeed ekpyrotic, there may be no Big Bang gravitational waves to observe, however subtle the instruments.

And so any talk of Swedish gold - Nobel prizes - for the first finder of a B-mode, and probably also for the proposers of inflation, is either spot on or way too premature. Lange, for one, has bets on the former. In the next few years, he will have bolometers of his devising in space, on a balloon and at the South Pole. But he is also steeling himself in case of the latter. When he gives talks about the search for B-modes, he always inserts pictures of people chasing geese. If the current generation of experiments fails to detect anything, he will humbly accept that Nature has put some things beyond his reach, and his lifetime. "Then, personally, I go do something else."

Eric Hand is a reporter for Nature based in Washington DC.

experiment will use a cryostat of liquid helium (far left) to cool instruments such as polarizationsensitive focal planes (near left) in its search for B-modes.
1. Wright, E. L. et al. Astrophys. J. 396, L13-L18 (1992)

2. Guth, A. H. Phys. Rev. D 23, 347-356 (1981).

3. Linde, A. D. Phys. Lett. B 175, 395-400 (1986)

4. Khoury, J., Ovrut, B. A., Steinhardt, P. J. \& Turok, N. Phys. Rev. D 64, 123522 (2001).

5. Bauman, D. et al. Preprint at http://arxiv.org/abs/0811.3919 (2009).

6. Hand, E. Nature doi:10.1038/news.2008.755 (2008).

7. Räth, C., Morfill, G. E., Rossmanith, G., Banday, A. J. \& Górski, K. M. Phys. Rev. Lett. 102,131301(2009)

\section{(1)}

